\title{
Information Analysis for Quantum Imaging Optimization
}

\author{
Alexander B. Mikhalychev ${ }^{1}$, Ilya L. Karuseichyk ${ }^{1}$, Svetlana V. Vlasenko ${ }^{1}$, Bänz Bessire ${ }^{2}$, \\ Dmitry A. Lyakhov ${ }^{3}$, Dominik L. Michels ${ }^{3}$, André Stefanov' ${ }^{2}$, Dmitri S. Mogilevtsev \\ 1. B.I. Stepanov Institute of Physics of NAS of Belarus, Nezavisimosti ave., 68-2, 220072 Minsk, Belarus \\ 2. Institute of Applied Physics, University of Bern, Sidlerstrasse 5, CH-3012 Bern, Switzerland \\ 3. Computer, Electrical and Mathematical Science and Engineering Division,4700 King Abdullah University of Science and Technology, \\ Thuwal 23955-6900, Kingdom of Saudi Arabia
}

Quantum imaging emerged quite recently as a breakthrough technology of overcoming the diffraction limit in microscopy and enhancement of optical resolution without the necessity to use hard radiation or perform scanning in the near field [1,2]. Both, the quantum imaging itself and the more "classical" techniques inspired by it (for example, super-resolution optical fluctuations imaging - SOFI [3]), rely on detection and analysis of photon (intensity) correlations. Typically, it is believed that the more correlated illuminating light is used and the higher order of the correlations is measured, the larger super-resolution can be achieved. That conclusion is based on efficient narrowing of the point-spread function, which, however, does not necessarily imply better resolution as the ability to reconstruct smaller features of the investigated object successfully.

More consistent and accurate quantification of the achieved resolution can be performed by using Fisher information (see e.g. Refs. [4-6]). The Cramér-Rao bound connects the errors of the object parameters reconstruction to the Fisher information matrix. Further, the resolution can be determined as the minimal size of the object features, for which the predicted reconstruction error does not exceed the threshold, specified by the experimenter. Minimization of the error values, provided by Cramér-Rao bound, and maximization of the achievable resolution give a clue for finding the optimal configuration of an imaging experiment.

In this contribution, we apply the information-based approach to optimization of several imaging schemes and present the obtained results, which are rather counter-intuitive [5,6]. First, as transition from a coherent illumination to spatially correlated biphotons or spatially incoherent light increases resolution, one could expect that usage of maximally correlated biphotons or maximally incoherent light would be the best choice. We show that the optimal transverse correlation (coherence) length is finite, in contrast to the anticipation of the zero value being optimal. Second, we analyse the dependence of the resolution in SOFI and thermal-light imaging on the correlations (cumulants) order. While an unlimited resolution increase could be expected with the growth of that order, there exists a finite optimal correlations order leading to a finite resolution limit even for an idealized theoretical model. Finally, we show that, being given an $n$-photon spatially entangled state, it is more profitable to measure $(n-1)$-photon correlations, rather than $n$-photon coincidences even in terms of the information gain per a single detection event.

(a)

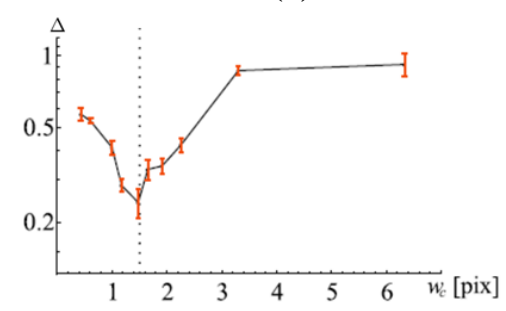

(b)

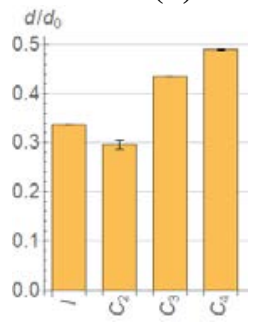

(c)

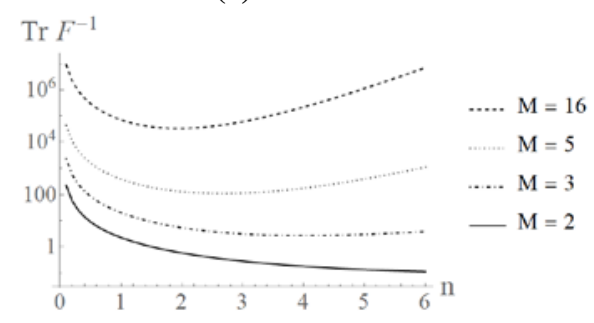

Fig. 1 Examples of imaging conditions optimization. (a) Dependence of the realized distance between the imaged object and the reconstruction result for pseudo-thermal light imaging on the transverse coherence length of the light for measured data [5]. The dashed line shows the information-based prediction of the optimal coherence length. (b) Dependence of the predicted resolution in SOFI, expressed in terms of Rayleigh limit $d_{0}$, on the used cumulant order. (c) Dependence of the predicted reconstruction error on the order of analyzed correlations $n$ for imaging of an object, which consists of $M$ pixels, for pseudo-thermal light imaging.

\section{References}

[1] Y. Shih. An introduction to quantum optics: photon and biphoton physics (CRC press, 2018).

[2] V. Giovannetti, et al., "Sub-Rayleigh-diffraction-bound quantum imaging,” Phys. Rev. A 79, 013827 (2009).

[3] T. Dertinger, et al., "Fast, background-free, 3D super-resolution optical fluctuation imaging (SOFI),” PNAS 106, 22287-22292 (2009).

[4] M. E. Pearce, et al., "Precision estimation of source dimensions from higher-order intensity correlations,” Phys. Rev. A 92, 043831 (2015).

[5] A. B. Mikhalychev, et al., "Efficient reconstructing compound objects by quantum imaging with higher-order correlation functions," Comm. Phys. 2, 134 (2019).

[6] S. Vlasenko, et al., “Optimal correlation order in superresolution optical fluctuation microscopy,” Phys. Rev. A 102, 063507 (2020). 